\title{
MicroRNA amplification and detection technologies: opportunities and challenges for point of care diagnostics
}

\author{
Vivek Priy Dave ${ }^{1,4} \cdot$ Tien Anh Ngo $\mathbb{1}^{1} \cdot$ Anna-Karin Pernestig ${ }^{2} \cdot$ Diana Tilevik $^{2} \cdot$ Krishna Kant $^{3} \cdot$ Trieu Nguyen $^{1} \cdot$ \\ Anders Wolff ${ }^{3} \cdot$ Dang Duong Bang ${ }^{1}$
}

Received: 17 May 2018 / Revised: 3 August 2018 / Accepted: 30 August 2018 / Published online: 12 December 2018

(c) United States \& Canadian Academy of Pathology 2018

\begin{abstract}
The volume of point of care (POC) testing continues to grow steadily due to the increased availability of easy-to-use devices, thus making it possible to deliver less costly care closer to the patient site in a shorter time relative to the central laboratory services. A novel class of molecules called microRNAs have recently gained attention in healthcare management for their potential as biomarkers for human diseases. The increasing interest of miRNAs in clinical practice has led to an unmet need for assays that can rapidly and accurately measure miRNAs at the POC. However, the most widely used methods for analyzing miRNAs, including Northern blot-based platforms, in situ hybridization, reverse transcription qPCR, microarray, and next-generation sequencing, are still far from being used as ideal POC diagnostic tools, due to considerable time, expertize required for sample preparation, and in terms of miniaturizations making them suitable platforms for centralized labs. In this review, we highlight various existing and upcoming technologies for miRNA amplification and detection with a particular emphasis on the POC testing industries. The review summarizes different miRNA targets and signals amplification-based assays, from conventional methods to alternative technologies, such as isothermal amplification, paper-based, oligonucleotide-templated reaction, nanobead-based, electrochemical signalingbased, and microfluidic chip-based strategies. Based on critical analysis of these technologies, the possibilities and feasibilities for further development of POC testing for miRNA diagnostics are addressed and discussed.
\end{abstract}

These authors contributed equally: Vivek Priy Dave and Tien Anh Ngo

Supplementary material The online version of this article (https:// doi.org/10.1038/s41374-018-0143-3) contains supplementary material, which is available to authorized users.

$\triangle$ Tien Anh Ngo

tinan@food.dtu.dk

1 Laboratory of Applied Micro and Nanotechnology (LAMINATE), Division of Microbiology and Production, National Food Institute, Technical University of Denmark, 2800 Kgs Lyngby, Denmark

2 Systems Biology Research Centre, School of Bioscience, University of Skövde, Skövde, Sweden

3 Department of Micro- and Nanotechnology, Technical University of Denmark, 2800 Kgs Lyngby, Denmark

4 Present address: Boditech Med Inc, 43 Geodudanji 1-gil, Dongnae-myeon, 24398 Chuncheon-si, Gangwon-do, South Korea

\section{Introduction}

MicroRNAs (miRNAs) are evolutionary conserved, $\sim 18-24$ nucleotides long non-coding RNA, playing a significant role in controlling human gene expression by post-transcriptional gene regulation or silencing [1]. Further, aberrant expression of a single miRNA can regulate the activity of multiple genes [2]. Previous studies have demonstrated that changes in miRNA expression contribute to a wide variety of human disease states and disorders such as cancer, cardiovascular, autoimmune, neurodegenerative, liver, and inflammatory diseases [3-5]. The miRNAs not only circulate in the human peripheral blood in a remarkably stable form [6-9], they are also widely present in other body tissues and fluids such as urine, saliva, milk, and cerebrospinal fluid [9-11]. These characteristics indicate that the miRNAs are potential biomarkers for diagnostic purposes. The miRNAs are involved in disease origin and development, and are pathology-specific, thus altered miRNA expression have been addressed for early detection and diagnostics, classification, prognostics, as well as predictive diagnostics. 
Fig. 1 Schematic demonstration of POC diagnostics. (a) Illustration of the routine laboratory diagnostics, (b) sites for POC tests (adapted from ref. [97]), and hierarchical feature for an ideal POC test/device (c) a

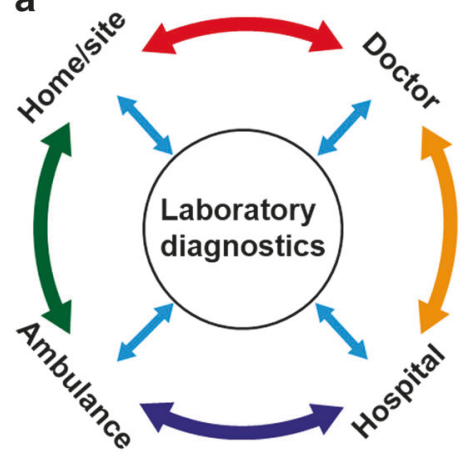

b

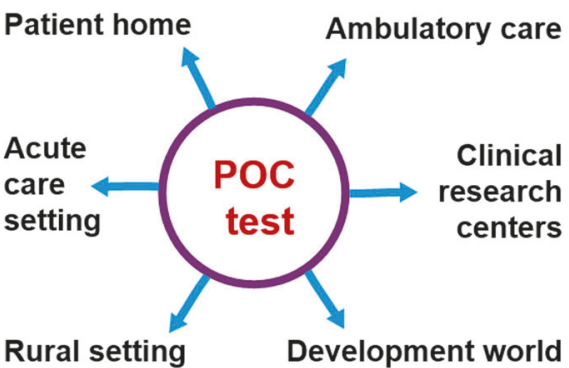

C

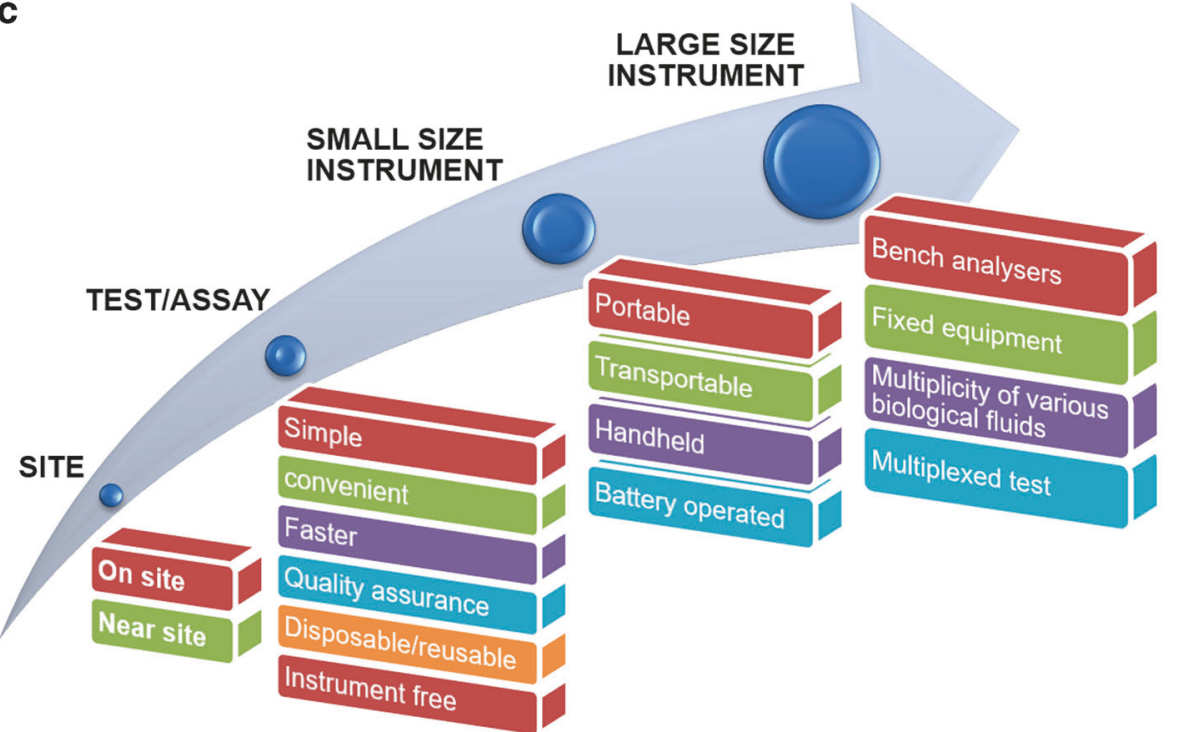

During the past decades, point of care (POC) diagnostics have become an integral part of the transformation of the healthcare landscape. In routine laboratory diagnostics, tests are usually performed in a laboratory setup, away from the individual patient (Fig. 1a). POC testing is defined as " $A$ testing that is performed near or at the site of a patient with quicker results leading to a possible change in the patient care" [12]. The use of POC testing is becoming more and more popular in clinical diagnosis due to its convenience, timeliness, and potential to improve the outcome for the patient. In this context, a POC test/device can be used in a patient's home, ambulatory care setup, acute care setup, and clinical research centers along with low resource rural settings (Fig. 1b). POC testing may reduce repeated hospital visits and time to wait for results for the patient. It may also help in immediate therapy decision and avoids follow-up appointments to discuss results [13]. In an ideal way, POC testing assay/device should be cheaper, faster, and smarter. However, it can be classified differently to define how close a POC test is to the ideal conditions (Fig. 1c). Up to date, many of the POC assays have been commercially available for detection or diagnosis of various clinical conditions.
These assays mainly rely on biochemical reactions, antigen-antibody interaction or nucleic acid hybridizations. Some examples include blood glucose testing, blood gas, and electrolytes analysis, pregnancy testing, infectious disease testing, etc.

Despite substantial advances in miRNA-related research, diagnostic tests based on miRNAs are not yet implemented in clinical routine. Thus, no standardized workflows have been established for analysis of miRNAs for the benefit of the patient. However, several promising miRNA-based assays exist that might eventually lead to routine applications, such as the development of a POC non-sputum-based test capable of detecting all forms of human tuberculosis [5, 14, 15]. These miRNA-based tests will be able to represent a significant improvement in turnaround time and accuracy for use in lower tiers of the healthcare system. In the cancer research area, miRNAs have perhaps gained the most interest as powerful tools for diagnostics as well as predicting the patient outcome [16-20]. The New York State Department of Health Clinical Laboratory Evaluation Program has approved a molecular test, based on Rosetta Genomics' miRNA technology, for clinical use [21, 22]. 
When using this system to test a sample of a patient's tumor, the test classifies squamous-cell carcinoma of the lung with a sensitivity of $96 \%$ and specificity of $90 \%$. Columbia University Medical Center developed and validated this assay. Food and Drug Administration (FDA) has already approved a handful of miRNA-based tests aimed at guiding therapeutic management. For instance, for the classification of cancer of unknown primary origin, for lung cancer type, for thyroid cancer stratification, and for breast cancer metastasis [23, 24]. Although the results are promising and may be used to guide decisions regarding treatment that leads to improved patient outcomes, this field is still in the infant stage, and the adoption of these tests by the clinical practice requires more clinical studies. Recent reviews have also emphasized the role of circulating miRNAs as potential and emerging biomarkers for diagnosis of human infectious diseases [5]. The increased amount of studies on miRNAs as promising diagnostic biomarkers has also been followed by a growing number of public databases providing information on the relationships between miRNA and diseases [25-27]. Thus, developing POC assays and diagnostics tools for detection of known miRNA holds a promising area of research in the coming decade. In this review, we will summarize and discuss the existing assays based on conventional methods for miRNA amplification and detection, and highlight upcoming technologies with a particular emphasis on the POC testing industries. Based on critical analysis of these technologies, the possibilities and feasibilities for further development of POC testing for miRNA diagnostics are addressed and discussed.

\section{Conventional miRNA detection methods}

Moving into the field of biomarkers and therapeutics, miRNA is a rising star. The relatively new field of miRNA has gained an accelerated growth regarding prognostic, diagnostic, functional, and therapeutic bioanalysis. Existing assays such as Northern blot (NB), in situ hybridization (ISH), reverse transcription qPCR (RT-qPCR), microarray, and next-generation sequencing (NGS) are, however, far from the ideal POC diagnostic assays. The advantages and disadvantages of these assays are summarized in Table 1.

\section{Northern blotting}

NB for miRNA analysis is a readily available technology for molecular biology laboratories. To perform an NB analysis, the RNA in a sample is size-separated through denaturing gel electrophoresis, transferred and cross-linked to a membrane and hybridized using a nucleic acid probe complementary to the target RNA. NB technique can be used to analyze the expression levels of individual miRNAs 
[28]. NB analysis has the advantage of simultaneously detecting the mature miRNAs and miRNA precursors, but it is expensive, very labor-intensive and time-consuming approach which also requires radio-labeling. In addition, NB is not typically used for quantitative analysis. It is considered to be semi-quantitative and providing information regarding relative RNA expression within a sample or across samples [29, 30]. For detection, both radioactive and non-radioactive probes can be used where labeling strategies include end labeling as well as uniform probe labeling [31, 32]. Due to its high sensitivity, radioactive labeling with ${ }^{32} \mathrm{P}$ is most commonly utilized. However, the health hazard, safety measurements, and special training associated with radiation use, as well as the short halflife of the probe are a significant disadvantage. While NB is widely used throughout the field, alternative techniques, such as RT-qPCR, nuclease protection assays, fluorescent in situ hybridization (FISH), and microarrays have advantages over it. Mainly, these techniques are considered to be more sensitive, and they also tend to be less sensitive to RNA degradation. For example, RTqPCR, nuclease protection assays, and FISH allow examination of multiple genes at once, and microarrays are high-throughput. FISH does not require the isolation of RNA. In addition, it provides information about RNA localization within the cell or tissue. Even though there are a number of disadvantages in NB, it is the only method that provides information regarding both sequence and length. For this reason, NB is still widely used for RNA detection and to validate results obtained with other methods.

\section{In situ hybridization}

ISH is a commonly used method to visualize expression and localization of molecules within a cell, tissue, or embryo. ISH techniques involve the use of labeled complementary nucleic acid probes to detect single-stranded DNA or RNA in tissue sections or fixed cells [33] and were used for detection of miRNA for the first time in 2006 [34]. The primary advantage of ISH in comparison to other miRNA detection methods is its ability to monitor the cellular and sub-cellular distributions, as well as to determine the spatiotemporal expression profile of miRNAs. This can be important to determine the spatiotemporal expression of miRNAs in elucidating their biological role as well as their pathologic involvement in numerous diseases. At present, ISH is the only technique for miRNA profiling that preserves RNA integrity and identifies the native locations of miRNA in a single cell, inside tissues, or in cell compartments [35]. RNA FISH is a cell-based technique for detecting mRNA transcripts. Recent advances in various methods for signal amplification and super-resolution imaging have enabled the development of single molecule RNA FISH (smRNA-FISH) techniques. Several approaches, including branched DNA probes, tyramide signal amplification, quantum dots (QDs), and padlock-rolling circle amplification (RCA), have been used for signal enhancement [36]. Although the low-throughput nature remains a major limitation of this technique, the recent development of directly labeled fluorescence probes has allowed this method to detect multiple miRNAs per reaction [37]. FISH technology has made significant progress with the innovation of novel labeling methods and the introduction of super-resolution imaging systems for fine mapping of intra-nuclear genomic structures and single cells single molecule profiling of cytoplasmic RNA transcription. Even with the introduction of genomic technologies, such as microarray analysis and exome sequencing, the FISH analysis still cannot be substituted in the field of genetic diagnosis [38-40]. Micro-fluidic devices for miniaturized and automatic FISH applications are currently under development [41-43]. Further research on the identification of more diseases specific probes and labeling methods will give strength to validate these platforms in diagnostics FISH POC settings.

\section{Reverse transcription qPCR}

RT-qPCR is a gold standard approach to quantify circulating miRNAs [44], for which there are some protocols and commercial assays such as miRCURY LNA qPCR from Exiqon, TaqMan assays from Applied Biosystems/Fisher Thermo Scientific, and two-tailed RT-qPCR miRNA assays from TATAA Biocenter. The principle of these RT-qPCR methods focuses on two steps: cDNA synthesis using RT followed by detection of amplified products using a conventional qPCR with either intercalating dye or TaqMan probe. Non-common sequence features are used for the enrichment and amplification due to the short length of target miRNAs. Additionally, the mature miRNA sequence is present in both pre-miRNAs and pri-miRNAs. Therefore, the design of miRNA primers in both steps is critical to obtain the specificity and sensitivity of RT-qPCR assays for detection of mature miRNAs. In the step of cDNA synthesis, target-miRNAs could be reverse transcribed by using universal [45-47] or specific RT primers [48-51] for highly accurate miRNA quantification. To utilize universal primers for RT, all 3'-end of miRNAs are first tailed to possess a common sequence, such as poly(A or $\mathrm{U}$ ) by using poly(A or $\mathrm{U})$ polymerase at its $3^{\prime}$-end as the pre-treatment step, and then reverse transcribed by a universal primer [45-47]. In this case, the RT primer is designed an oligo ( $\mathrm{dT}$ or $\mathrm{dA}$ ) sequence at $3^{\prime}$-end and a universal primer-binding sequence at $5^{\prime}$-end that is used to amplify the target sequence cDNA in the qPCR step. To reduce the pre-treatment step above, 
the target miRNAs could be reverse transcribed directly into cDNA by using specific primers, such as stem-loop RT primers [48], linear RT primers [49], DNA pincer probe [50], and two-tailed RT primer [51]. By designing a specific probe, this method can specifically quantify mature miRNAs from their precursors, and discriminate miRNA homologs from the same miRNA family. Recently, Androvic et al. have developed a novel method based on two-tailed RT primer for highly accurate miRNA quantification, namely two-tailed RT-qPCR [51]. In this method, the stem-loop RT primers are designed to have two hemiprobes at both $3^{\prime}$-end and $5^{\prime}$-end that precisely hybridize to two different parts of the miRNA. The method demonstrates a dynamic range of seven logs, high sensitivity with a detection limit of 10 targeted miRNA molecules. The method can discriminate between similar miRNAs, and ability to quantify iso-miRNAs [51].

In an alternative approach, $\mathrm{Li}$ et al. developed an RT-free qPCR based on enzymatic ligation of DNA stem-loop primers for miRNA detection, in which a pair of stem-loop primers is designed to hybridize with the target miRNA [52]. The resulting joint DNA is then used as a template for the PCR amplification. This method is a simple, accurate, and inexpensive qPCR for miRNAs detection. However, the method is limited by low ligation efficiency and low sensitivity [52] compared to RT-qPCR assays [53].

It is known that RT-qPCR is a powerful tool to identify miRNAs. However, this technique is time-consuming, complicated, and requires sophisticated, large, and expensive thermal cycling equipment for amplification and quantification and is not suitable to apply for POC testing. Developing a portable, easy-to-use miRNA amplification detection system with sufficient selectivity and sensitivity for POC diagnostics is not a simple task. A portable PCR system requires temperature cycling, which is a major battery drainer. Moving towards POC, isothermal miRNA amplification may serve as a promising technique, which we will discuss in the next section. Another challenging is micro-fluidics. Transportation of the PCR reaction from the PCR chamber into the microarray plate in a portable device requires the timely managing of a few microliters over a few millimeters. Dispensing the rinsing solutions and all reagents in a timely fashion complicates the design further. Finally, the problem of the detection system, which requires highly sensitive charge-coupled device (CCD) cameras that are big-sized, expensive, requiring cooling, and sophisticated electronics. Another possibility is the use of photodiode arrays [54]. Photodiodes are small, very sensitive, and simple to operate photodetectors capable of transforming light into either current or voltage. Taking collective measurements on the challenges, RT-qPCR could be a significant technology for the miRNA POC testings shortly.

\section{Microarrays}

Microarray-based methods are widely used for gene expression analysis studies and were among the first technologies to be utilized for parallel analysis of a vast number of miRNAs. Microarrays rely on nucleic acid hybridization between target molecules and their corresponding complementary probes. Several technical variants of the approach have been developed independently in the past few years, including the probe design, immobilization chemistry, sample labeling, and microarray chip signaldetection methods $[55,56]$. The strength of microarraybased approaches is their ability to quantify large numbers of miRNAs simultaneously in a single experiment. Microarrays are often chosen for identification and validation of novel miRNA signatures and are still one of the most widely used as high-throughput methods for detecting miRNA levels [57]. Measurement of differential expression of various miRNAs is crucial to determine the physiological and pathophysiological status of cellular microenvironment. In this context, GeneChip miRNA Array (Thermo Fisher Scientific) is one of the well-known platforms for studying and deciphering the role of miRNAs. This array has comprehensive coverage designed to interrogate all mature miRNA sequences in miRBase Release-20, while only requiring a low sample input of $130 \mathrm{ng}$ total RNA. The high-resolution automated GeneChip Scanner 3000 7G has significantly improved the miRNA applications. It fits easily into a benchtop environment. Its solid-state laser eliminates the need for an external laser power supply or a special cooling system under the bench. Target preparation on the Nimbus instrument helps minimize run-to-run variability and the labor burden associated with complicated manual pipetting. Despise all the facts; this platform is limited to a centralized lab or research lab as much more miniaturization is required to move these platforms to POC settings.

\section{Next-generation sequencing}

Sequence-based methods for miRNA profiling determine the nucleotide sequence of miRNAs and involve RNA isolation, ligation of linkers to the $3^{\prime}$ and $5^{\prime}$-ends, RT, and PCR amplification. Traditionally, sequence-based methods were time-consuming and expensive methods for miRNA detection [58]. Since 2007, NGS instruments that can process millions of sequence reads in parallel in a few days have driven the discovery of most miRNAs [59]. In comparison with other high-throughput methods, such as microarray, the NGS technologies do not suffer from problems like background noise and cross-hybridization. The NGS also offers other significant advantages such as the possibility to generate comprehensive and definitive analyses of all miRNAs in samples, including those derived 
from sera and plasma [60]. The techniques do not require knowledge of target miRNAs or specific probes or primers, and it can, therefore, detect both known and unknown miRNAs. NGS is the best platform for miRNA discovery since unambiguous libraries of miRNAs are generated from humans and other organisms [61, 62]. However, the technique has some limitations: (1) NGS is still too expensive for routine laboratory work and (2) the technique requires computational infrastructure for data analysis and interpretation. It is therefore not yet suitable for POC testing. Companies within the space of personalized health care are designing instruments to target specific needs across the clinical diagnostic spectrum. Therefore, they are adding NGS capabilities to their portfolio. As long as the genomic era interfaces with personalized medicine, NGS uptake will continue. Several NGS companies, such as Genapsys, Gnubio (acquired by Biorad), and Genia (acquired by Roche), have innovative technologies in the pipeline with a focus on $\mathrm{iPad} /$ chip-sized devices using semiconductor technology providing results with a short turnaround time. Such tests will find their place clinics, and hence NGS will soon be offered in clinical settings. Further POC mode of sequencing will provide simplified handling, enable fast accurate results, reduce sample volumes/reagent and produce minimal waste without the need of expertize.

\section{miRNA amplification and detection methods for POC diagnostics}

Each of the assays described in the previous section has its own individual limitations (Table 1). Although NB is easy to perform, it is laborious and time-consuming. Furthermore, it requires radio-labeling, which can introduce significant contamination and has low detection efficiency. RT-qPCR and microarrays have good detection limits, but the requirement of equipment, such as thermocycler and a scanner, is not affordable by small and medium scale clinics, thus limiting their use. The development of methods with highly efficient and low-cost are needed due to the limitations mentioned in the current existing assays. Keeping in view the fact of limitations, the concept called lab-ona-chip (LOC) may become the dominant miRNA POC testing technology in the future. The development of LOC, sometimes referred to microchip, is on the way to grow from the microelectronics industry through techniques of miniaturization and micro-fabrication. Such devices have been defined as ones that perform analysis at microscopic scales, i.e., $1-500 \mu \mathrm{m}$ and incorporate microfilters, microchannels, microarrays, micropumps, microvalves, and bioelectronics chips [63]. Thus, these core technologies may play a pivotal role in the foundation of POC testing of miRNA. In addition, more attention is currently paid to alternative amplification and detection technologies at the POC industries (Table 2). A common theme of these newly developed methods is a combination of a multi-step-signal amplification with some sensitive signal output unit to achieve excellent detection efficiency for on-site or near-site POC miRNA diagnostics.

\section{Isothermal amplification-based assays}

Isothermal amplification has emerged as a robust method for quantification of nucleic acids and has already proven its utility for developing highly specific and sensitive miRNA assays. Compared to RT-qPCR, isothermal amplification can be performed without precise control of temperature cycling and is well fitted for detecting short RNA or DNA. Isothermal amplification can rapidly and efficiently amplify the target nucleic acids or the signal of a recognition event at constant temperature under simple conditions. A large family of isothermal amplification techniques has successively been developed, such as RCA, exponential amplification reaction (EXPAR), hybridization chain reaction (HCR), catalytic hairpin assembly (CHA), strand-displacement amplification (SDA), duplex-specific nuclease signal amplification (DSNSA), and loop-mediated isothermal amplification (LAMP). These methods mainly rely on enzyme-based replication, digestion, or enzyme-free strand displacement processes.

Jia et al. presented a typical example of the isothermal amplification of miRNAs, where the target-miRNA was designed to be the trigger of the isothermal amplification [64]. The template was designed to contain two identical segments: (1) complementary to the target-miRNA and (2) adjacent to the nicking site, with one segment upstream and one segment downstream. With this design, not only extension and cleavage occur at the nicking site, but the released sequence could also serve repeatedly as the trigger. By this way, the target-miRNA is exponentially amplified. Using a fluorescence dye-SYBR Green I, detection could occur in real-time (Fig. 2a). To further simplify the detection system, the authors [65] developed another detection based on the LAMP amplification [66], which only contained one single enzyme - the Bst DNA polymerase. Along with its DNA polymerase activity, the Bst enzyme also displays RNA polymerase (using a DNA template) and strand displacement activities. The loop-stem-like extension products could be detected using SYBR Green I. With repetitive strand extension and displacement, the stem-loop DNA products are further amplified. Here, the target miRNA also contributed to triggering the amplification process. In this method, the design of the templates and primers are complicated, and one set of probes is suitable for use with only one target. These factors increase the 


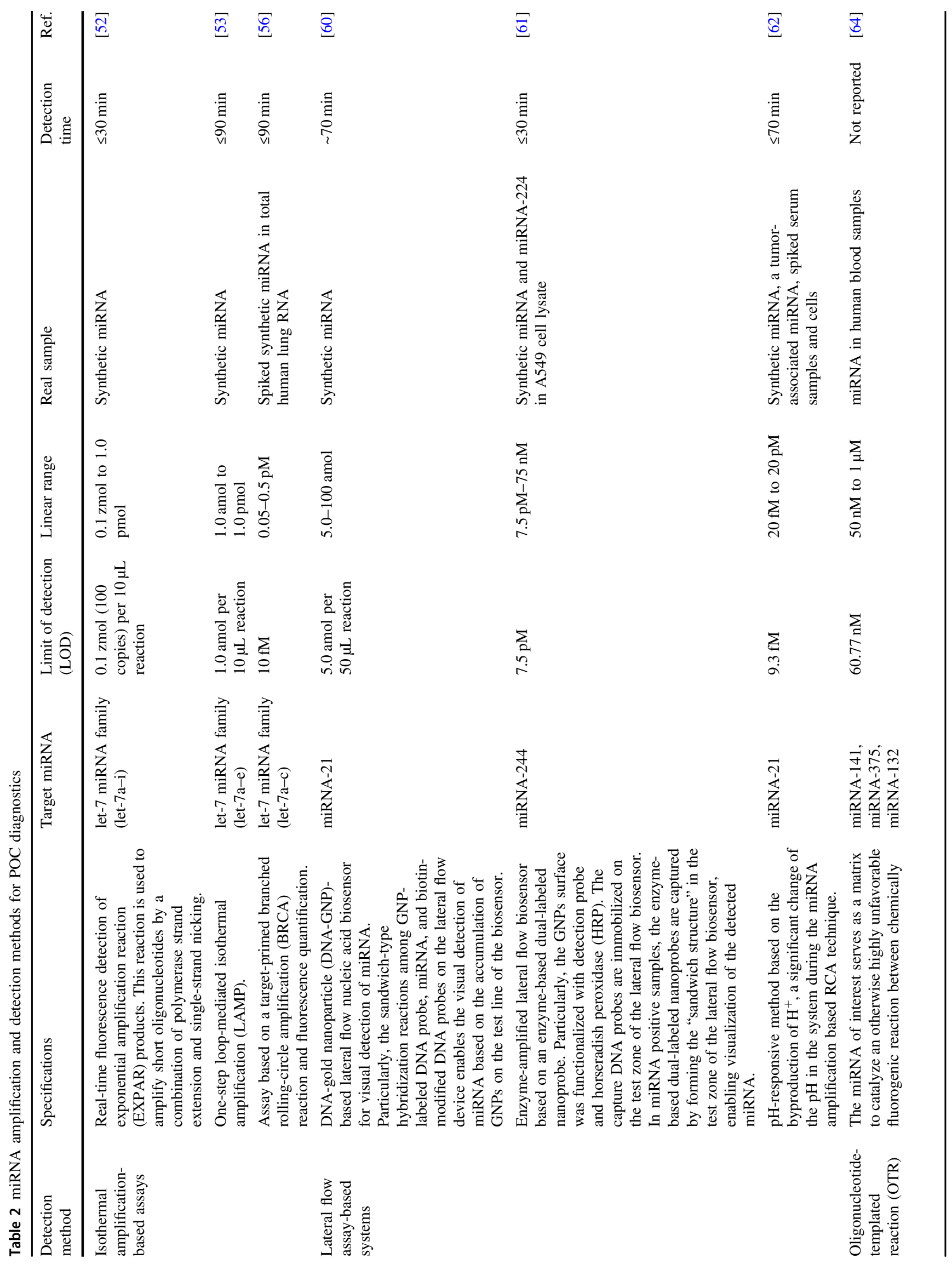




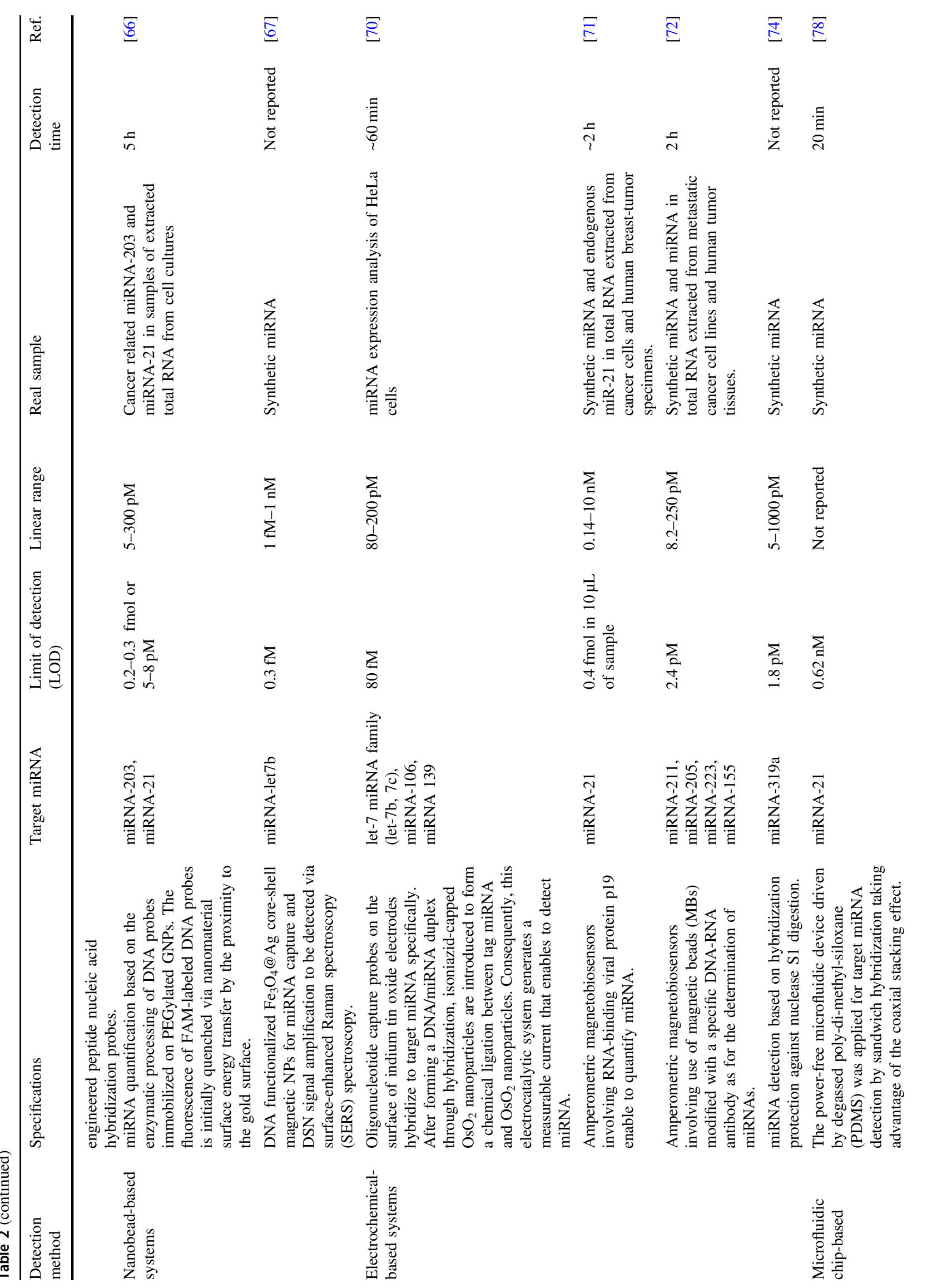




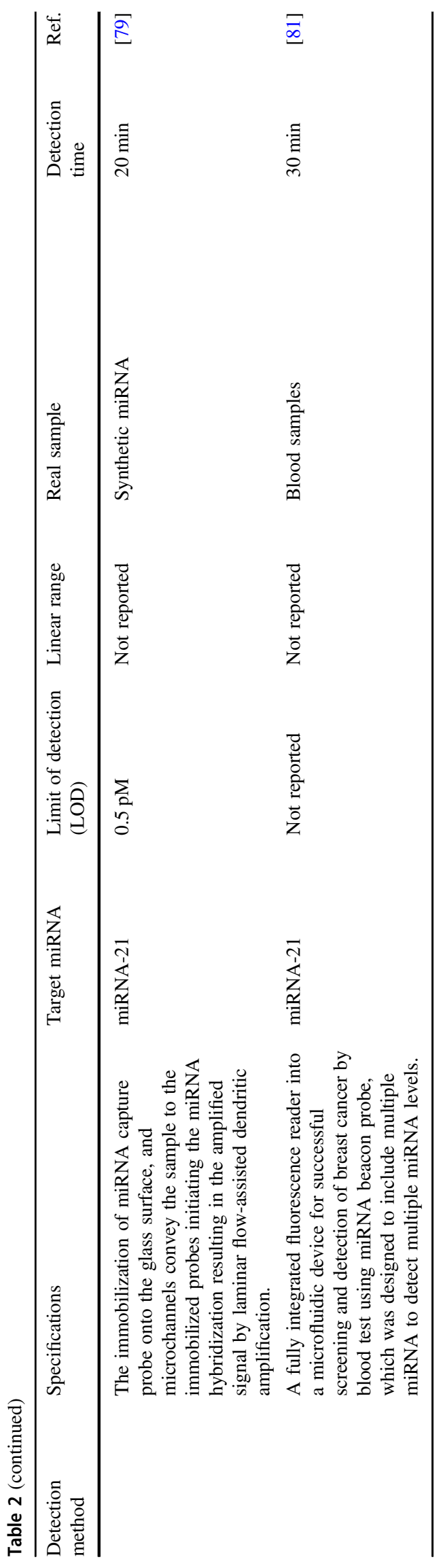

complexity and cost, especially when considering the application of this method in POC diagnostics (Fig. 2b).

RCA is a site-anchored isothermal nucleic-acid signal amplification. The reaction is based on the rolling circle replication mechanism. It was first applied to detect miRNA by Jonstrup and co-workers [67]. Subsequently, an improved design, called branched rolling-circle amplification (BRCA) (Fig. 2c) was developed [68]. This method is well-established and widely used for the specific and sensitive detection of short nucleotide sequences, and several subsequent improvements are made on its basis [69]. However, through this method, a single nucleotide mismatch at the middle position could easily be distinguished, but a terminal mismatch would lead to poor discrimination.

\section{Lateral flow assay (LFA)-based systems}

LFA-based POC devices are one of the most favorable approaches used for detection of target molecules of interest [70, 71]. The method provides a visual response usually based on the immune-chromatographic concept. LFA technology possesses many advantages due to its low operational cost, simple instrumentation, rapidity, and one step analysis, user-friendly format, high specificity and sensitivity, fewer interferences due to chromatographic separation, and portability [70, 71]. Additionally, the LFA paper-based devices use capillary force enabling usage without any external power. The white background of the paper also allows colorimetric and fluorescent-based detection. Furthermore, different types of labels, including gold, silver, and selenium nanoparticles (NPs), QDs, upconverting phosphors, fluorescent, and luminescent materials are utilized as visualizing markers for developing LFAs with improved sensitivity of detection. The size of all these diagnostic substances is adjustable for optical properties to develop specific LFA-based detection systems [71]. Recently, several lateral flow strip biosensors have been developed to detect many different targets, such as DNAs, mRNAs, proteins, biological agents, and chemical contaminants [70, 71]. Nevertheless, there are very few reports on the use of lateral flow strip biosensors for miRNA analysis. Fig. 3a represents generalized strategies to detect miRNAs using the LFA.

Hou et al. developed DNA-gold nanoparticle (DNA-GNP)-based lateral flow nucleic acid biosensor for visual detection of miRNA-21 [72]. They have used sandwich-type hybridization reactions among GNP-labeled DNA probe, miRNA-21 and biotin-modified DNA probes on the lateral flow device (Fig. 3b). The accumulation of GNPs on the test line of the biosensor enables the visual detection of miRNA-21. To avoid non-specific signals, a mung-bean nuclease, which catalyzes the degradation of the capture probe in miRNA negative samples, was used if 


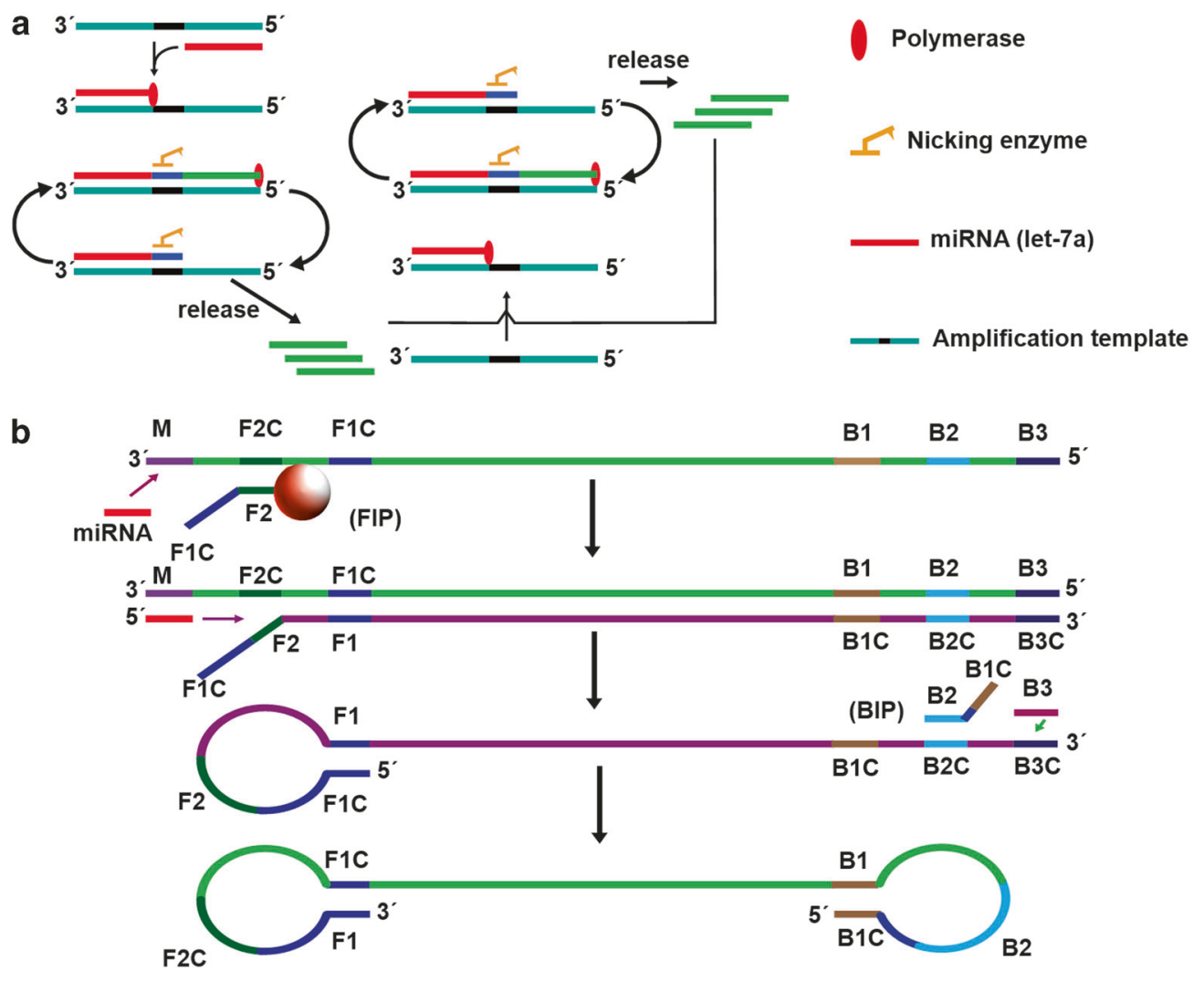

Bst DNA polymerase large fragment

C

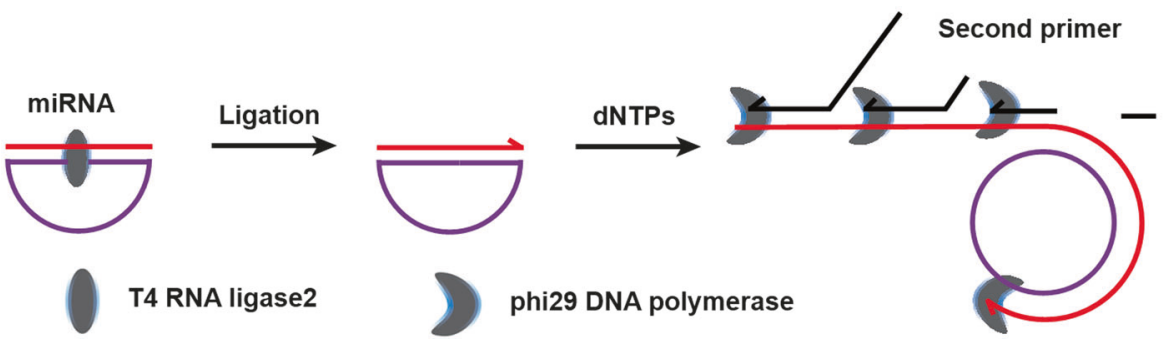

Fig. 2 Approaches based on Isothermal amplification. a Schematic representation of the exponential amplification reaction using let-7a miRNA as the amplification template. $\mathrm{P}$ indicates a phosphate group (adapted from ref. [64]). b Illustration of the LAMP reaction initiated

there is no tested miRNA in the samples. Gao et al. also reported an enzyme-amplified lateral flow biosensor based on an enzyme-based dual-labeled nanoprobe for visual detection of miRNA-224 [73]. In this method, the GNPs surface was functionalized with detection probe and horseradish peroxidase (HRP). The capture DNA probes were immobilized on the test zone of the lateral flow biosensor. In miRNA positive samples, the enzyme-based dual-labeled nanoprobes are captured by forming the "sandwich structure" in the test zone of the lateral flow biosensor, enabling visual detection of miRNA-224 (Fig. 3c) by $\mathrm{TMB} / \mathrm{H}_{2} \mathrm{O}_{2}$ enzymatic substrate reaction in the test zone [73]. Recently, Feng et al. have developed a by the target miRNA let-7a. Reproduced with permission from ref. [65]. c Illustration of the target-primed branched rolling circle amplification reaction. Reproduced with permission from ref. [68]

pH-responsive miRNA amplification method to amplify and quantify miRNA-21 in cancer cells just by using a pH test paper [74]. The operation is easy, and no other costly instrument is involved, making the method very attractive. The developed assay exploits the properties of highly efficient isothermal amplification of miRNA based on RCA technique. Large amounts of $\mathrm{H}^{+}$are produced as a byproduct of the amplification inducing significant changes in $\mathrm{pH}$, which can be monitored directly using a $\mathrm{pH}$ test paper or pH-sensitive indicators (Fig. 3d). The degree of color changes depends on the amount of miRNA, making it possible for quantitative analysis. The results agreed well with that of RT-qPCR analysis. 
Fig. 3 Approaches based on Lateral flow assay. a

Generalized strategies to detect miRNAs using the lateral flow assay (LFA). b Schematic description of miRNA detection using lateral flow nucleic acid strips with gold nanoparticles (adapted from ref. [72]).

c Schematic diagram of the hybridization reaction between the miRNA-capture probedetector followed by enzymemediated signal enhancement of the lateral flow strip (adapted from ref. [73]). d The principle of the $\mathrm{pH}$-responsive NRCAbased colorimetric assay for detection of miRNA followed by the equation of the reaction catalyzed by Bst DNA polymerase. The color changes of the $\mathrm{pH}$-responsive indicators under different $\mathrm{pH}$ (figure adapted with permission from ref. [74]) a
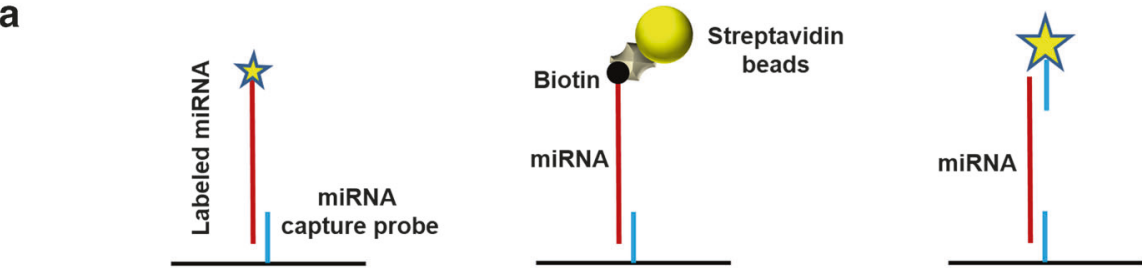

b

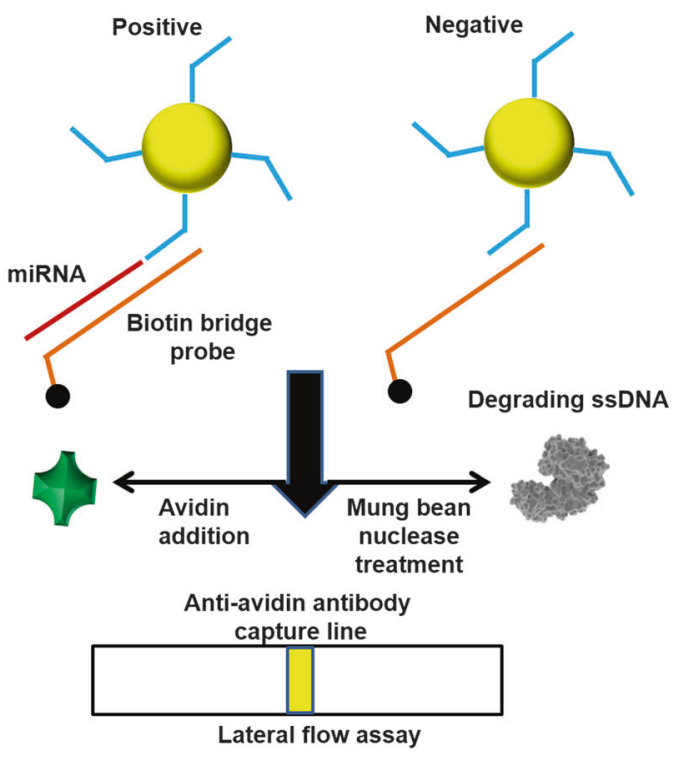

c

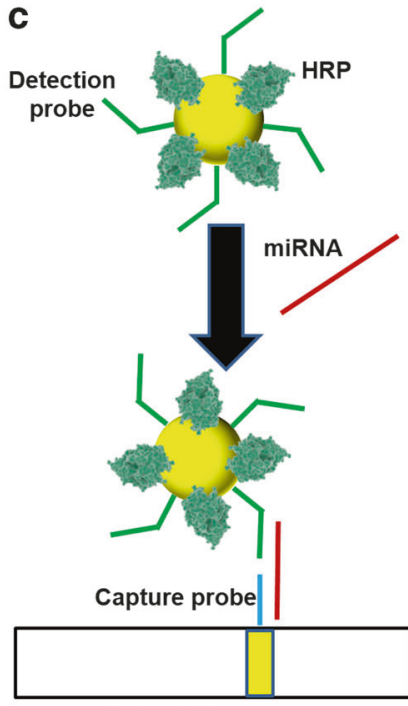

Lateral flow assay

d

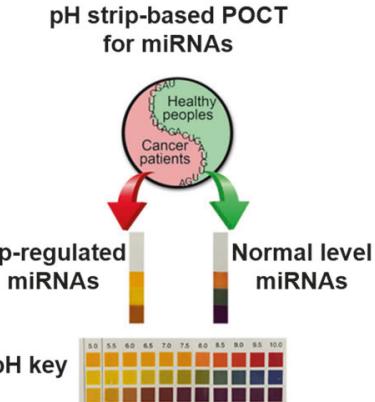

\section{Oligonucleotide-templated reaction}

Oligonucleotide templated reactions (OTRs) are very dynamic and have been successfully applied to a broad range of chemistries. The OTRs concept relies upon sequence-specific Watson-Crick base-pairing to bring together two reactive moieties or probe-heads, each attached to the end of an oligonucleotide. Because of their intrinsic specificity and high programmability, OTRs have found valuable applications in controlled organic synthesis, DNAencoded chemistry for nucleic acid sensing, both in vitro and in vivo. For sensing applications, OTRs have been engineered whereby only the nucleic acid target of interest acts as a template to catalyze, which can be monitored optically. Metcalf et al. have recently reported a novel sensing strategy based on OTRs [75] where only the
miRNA of interest serves as a matrix to catalyze an otherwise highly unfavorable fluorogenic reaction between chemically engineered peptide nucleic acid (PNA) hybridization probes (Fig. 4a) [76]. This method enables the quantitative detection of endogenous concentrations of circulating miRNA biomarkers, such as miR-375, miR-141, and miR-132, in blood samples and do not require any amplification step [76]. The process is isothermal and highly cost-effective.

\section{Nanobead-based systems}

Several innovative approaches based on the unique properties of nanostructured materials and devices have appeared in the scientific literature to support the widespread adoption of miRNA [77]. Typically, nanobead-based 
a

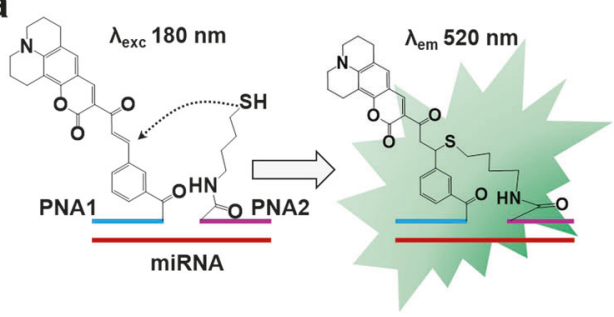

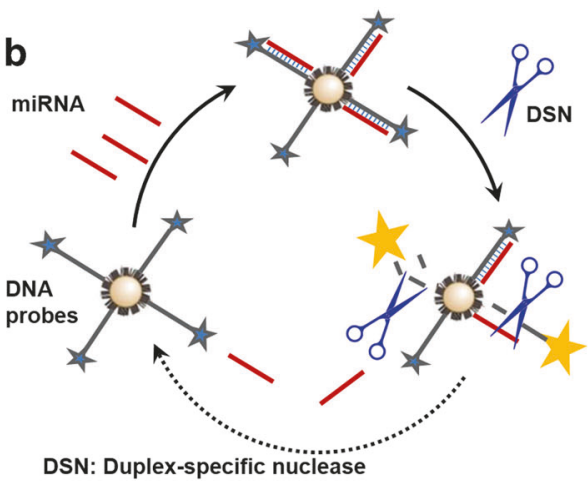

DSN: Duplex-specific nuclease

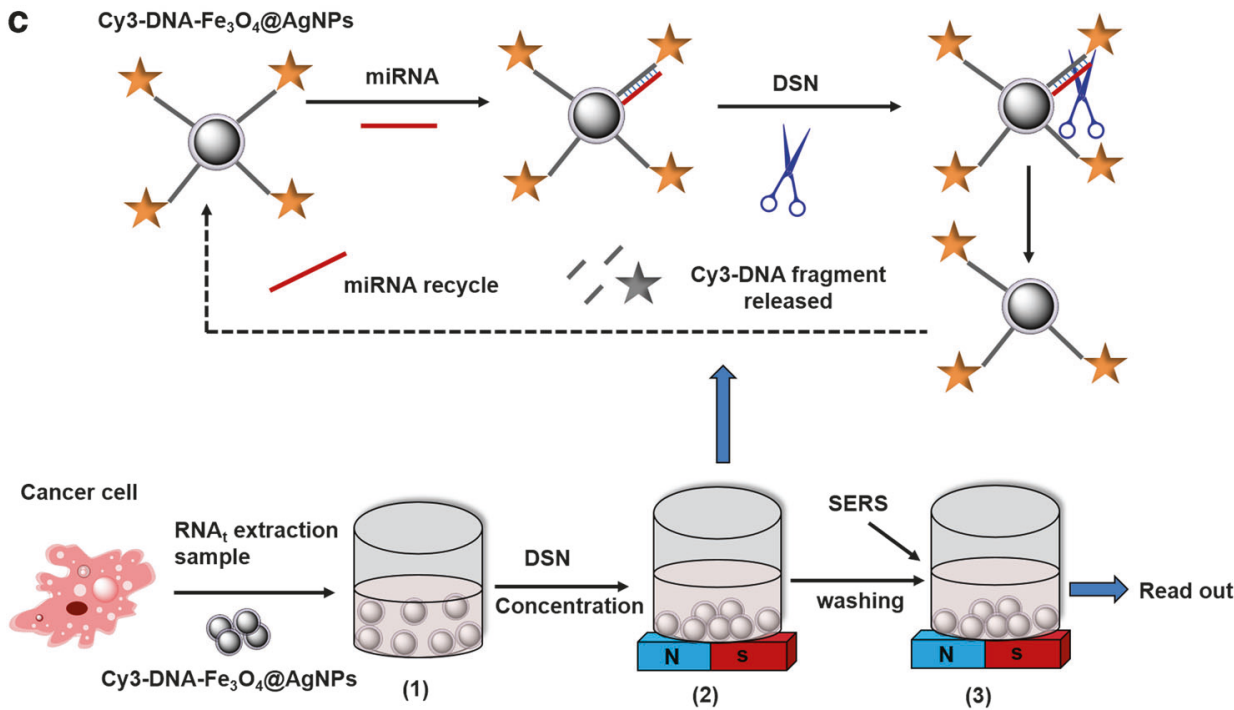

Fig. 4 Approaches based on oligonucleotide-templated reaction and nanobead. a Complementary Watson-Crick base-pairing between the RNA target and two engineered PNA probes catalyzes oligonucleotide-templated reaction where a fluorogenic Michael addition reaction and unleashes the quenched fluorescence of a coumarin derivative. Adapted with permission from ref. [76]. b DSN is a highly stable, nonspecific endonuclease, which possesses a strong preference for double-stranded DNA and DNA in DNA-RNA heteroduplexes. The substrate specificity of this enzyme is ideal for the

miRNA detection systems exploit the physiochemical properties of nanostructure materials, which induce transduction mechanisms after hybridization of target miRNA with the capture probe coupled nanobeads leading to quantifiable signals. In this context, several nano-particulate materials have excellent optical properties making them ideally suited for the development of sensing strategies. Some NPs are bright and stable fluorescence emitters, e.g., silver nanoclusters (AgNCs) and QDs, and can be used either directly or in fluorescence resonance energy transfer strategies. Other NPs, such as GNPs and carbonaceous NPs, can be used as efficient fluorescence quenchers in fluorescence recovery approaches.

Degliangeli et al. reported a real-time fluorescence recovery strategy for miRNA quantification based on the development of miRNA sensing strategies. Since target-miRNA remains intact during this process, under conditions of effective enzymatic activity, target-recycling amplification leads to significant signal enhancement. Adapted with permission from ref. [77]. c Schematic illustration of miRNA capturing using magnetic nanoparticles where mix RNAt sample with Cy-3-DNA modified $\mathrm{Fe}_{3} \mathrm{O}_{4} @ \mathrm{Ag}$ NPs concentrate samples by a magnet and add DSN for incubation after wash away the Cy-3-DNA fragments and read out Raman signal (adapted from ref. [79])

enzymatic processing of DNA probes immobilized on PEGylated GNPs [78]. The fluorescence of FAM-labeled DNA probes is initially quenched via nanomaterial surface energy transfer by the proximity to the gold surface. Upon hybridization with target miRNA, DNA:miRNA heteroduplexes are formed and become a substrate for the enzyme duplex-specific nuclease (DSN), which selectively cleaves the DNA strand leaving the miRNA untouched, resulting in target recycling amplification. The DNA-probe hydrolysis results in fluorescence recovery due to the release of the fluorophores in solution (Fig. 4b). Pang et al. also proposed DNA functionalized $\mathrm{Fe}_{3} \mathrm{O}_{4} @ \mathrm{Ag}$ core-shell magnetic NPs for miRNA capture and DSN signal amplification to be detected via surface-enhanced Raman spectroscopy (SERS) [79]. The detection occurs in a separate step following 
magnetic separation of the DSN-treated $\mathrm{Fe}_{3} \mathrm{O}_{4} @ \mathrm{Ag}$. The presence of target-miRNA produces an attenuation of the SERS signal (Fig. 4c).

\section{Electrochemical-based systems}

Electrochemical methodologies for miRNA detection have been developed to substitute classical methods [80]. Typically, an electrochemical genosensor-based hybridization [81] is established from two main components: (1) an electrode tethered short, single-stranded DNA/RNA probe, which can specifically hybridize with the target sequences, and (2) an electroactive hybridization indicator that translates the hybridization signal into the measurable current (amperometry, voltammetry) or charge accumulation (potentiometry) (Fig. 5a). For the first generation of miRNA electrochemical sensor, Gao and Yang developed a system (Fig. 5b) in which the oligonucleotide capture probes on the surface of indium tin oxide electrodes hybridize to target miRNA specifically [82]. After forming a DNA/miRNA duplex through hybridization, isoniazid-capped $\mathrm{OsO}_{2} \mathrm{NPs}$ are introduced to form a chemical ligation between tag miRNA and $\mathrm{OsO}_{2}$ NPs. Consequently, this electrocatalytic system generates a measurable current that enables detection of miRNA at the femtomolar level. Following this principle, some modified miRNA electrochemical sensors have been developed for the specificity and sensitivity of a wide range of miRNA detection [80].

In 2014, Pringarrón et al. reported another version of miRNA electrochemical biosensor platform, namely the amperometric magnetosensor [83], enabling the detection of a synthetic target-miRNA (miRNA-21) at $0.4 \mathrm{fmol}$ in raw samples without any amplification, preconcentration, and purification for diagnosis of various human cancers. In this system, the RNA-binding viral protein p19 immobilizedchitin magnetic bead was utilized as the biorecognition receptor for capturing target miRNAs/anti-miRNA duplex selectively. Particularly, a biotinylated antimiRNA-21 probe was introduced to hybridize to the miRNA-21 target specifically. The resulting miRNA-21/biotinylated antiRNA-21 duplex was then labeled with streptavidin protein conjugated to HRP for measurement of the catalytic current in the presence of $\mathrm{H}_{2} \mathrm{O}_{2}$ and hydroquinone (HQ) at a disposable screen-printed carbon electrode (SPCE) (Fig. 5c). Instead of viral protein p19, the specific antibodyfunctionalized magnetic bead was used as the bioreceptor for surface-confined DNA/RNA duplexes to develop a second generation of amperometric magnetosensor for detection of target miRNAs in clinical samples with a detection limit of 60 amol [84]. Similar to the first version, a biotinylated DNA probe was precisely and efficiently hybridized to the target-miRNAs in solution. The formation of DNA/miRNA duplex was then captured by a specific
DNA-RNA antibody/protein G-functionalized magnetic beads and detected amperometric on the SPCE in the present of $\mathrm{H}_{2} \mathrm{O}_{2} / \mathrm{HQ}$ (Fig. 5c).

Zhou et al. also reported a simple electrochemical biosensor for miRNA detection based on hybridization protection against nuclease S1 digestion [85] (Fig. 5d) [86]. The principle of this method is based on the change of the electrochemical response in the absence or present of specific hybridization process between the target-miRNA to DNA probe on GNPs/Au surface of the electrode when using nuclease $\mathrm{S} 1$ and redox probe of $\mathrm{Fe}(\mathrm{CN})_{6}{ }^{3-/ 4-}$. It is known that electrochemical methods have demonstrated a variety of advantages, such as specificity, sensitivity, simplicity, portability, as well as a low cost which makes these methods suited for POC diagnostics [87]. By using these approaches, miRNA can be directly detected in the clinical samples without any amplification.

\section{Microfluidic chip-based}

In the last two decades, LOC technology was the main era for microfabrication researchers and the LOC technology drew significant interest from the industries for its biomedical applications. The major advantages of LOC platforms are cost-effectiveness, short processing time, and multiplexed detection of the biological sample. These qualities of LOC attract researchers to develop advanced technologies and bring these technologies out of laboratory bench-top to the pocket of end users as POC system. It is also important to mention that micro-total analysis systems (MicroTAS) and micro-electro-mechanical systems (MEMS) play an equally important role in the development of POC system by providing a miniaturized electronic readout for such devices [88, 89]. In the development of POC system for miRNA detection, Arata et al. were first to develop a powerfree microfluidic device for detection of miRNA from a small sample volume (Fig. 6a) [90]. The device is driven by energy that is stored in degassed poly-di-methyl-siloxane (PDMS) in advance, eliminating the need for external power sources for fluid pumping. In addition, using an approach called laminar flow-assisted dendritic amplification (LFDA), the signal amplification is achieved onto the microfluidic device [91]. The miRNA is detected by sandwich hybridization technique, where a miRNA capture probe is immobilized onto the glass surface, and microchannels convey the sample to the immobilized probes. The miRNA hybridization takes place, and the signal is amplified by LFDA (Fig. 6b). Together with the advantages of self-reliance, this device might contribute substantially to the future development of miRNA POC diagnostics. Zhang et al. demonstrated a hand-powered electricity-free centrifugal microfluidic platform with sample multiplexing capability using molecular label detection to identify 
Fig. 5 Electrochemical-based approaches. a A schematic illustration of an electrochemical sensor for miRNA detection. b Representation of miRNA assay using electrocatalytic nanoparticles tags. Reproduced with permission from ref. [82] copyright 2006, Amerian Chemical Society. (c, left) Schematic demonstration of the p19-based amperometric magnetosensor designed for the determination of miR-21.

Reproduced with permission from ref. [83] copyright 2014 WILEY-VCH Verlag GmbH \& Co. KGaA, Weinheim. (c, right) Illustration of the antibodyfunctionalized magnetic beadbased amperometric magnetosensor designed for the determination of miRNAs. Reproduced with permission from ref. [84] Copyright 2016, American Chemical Society. d Schematic illustration of the detection strategy for microRNA assay based on hybridization protection against nuclease $\mathrm{S} 1$ digestion (adapted from ref. [86]) a

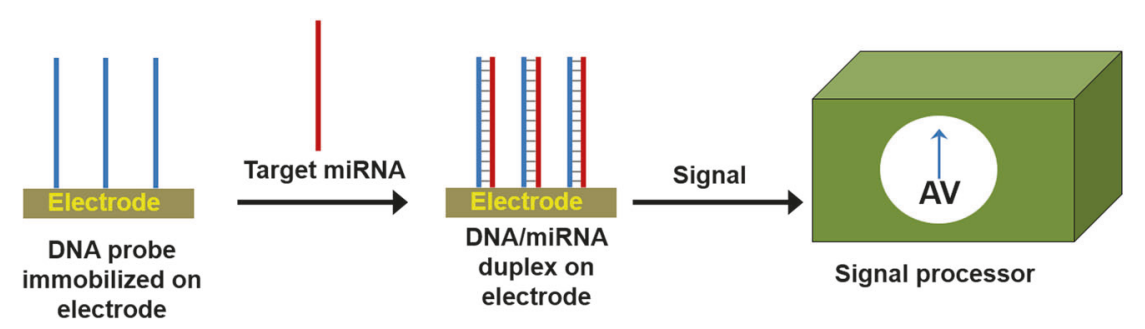

b
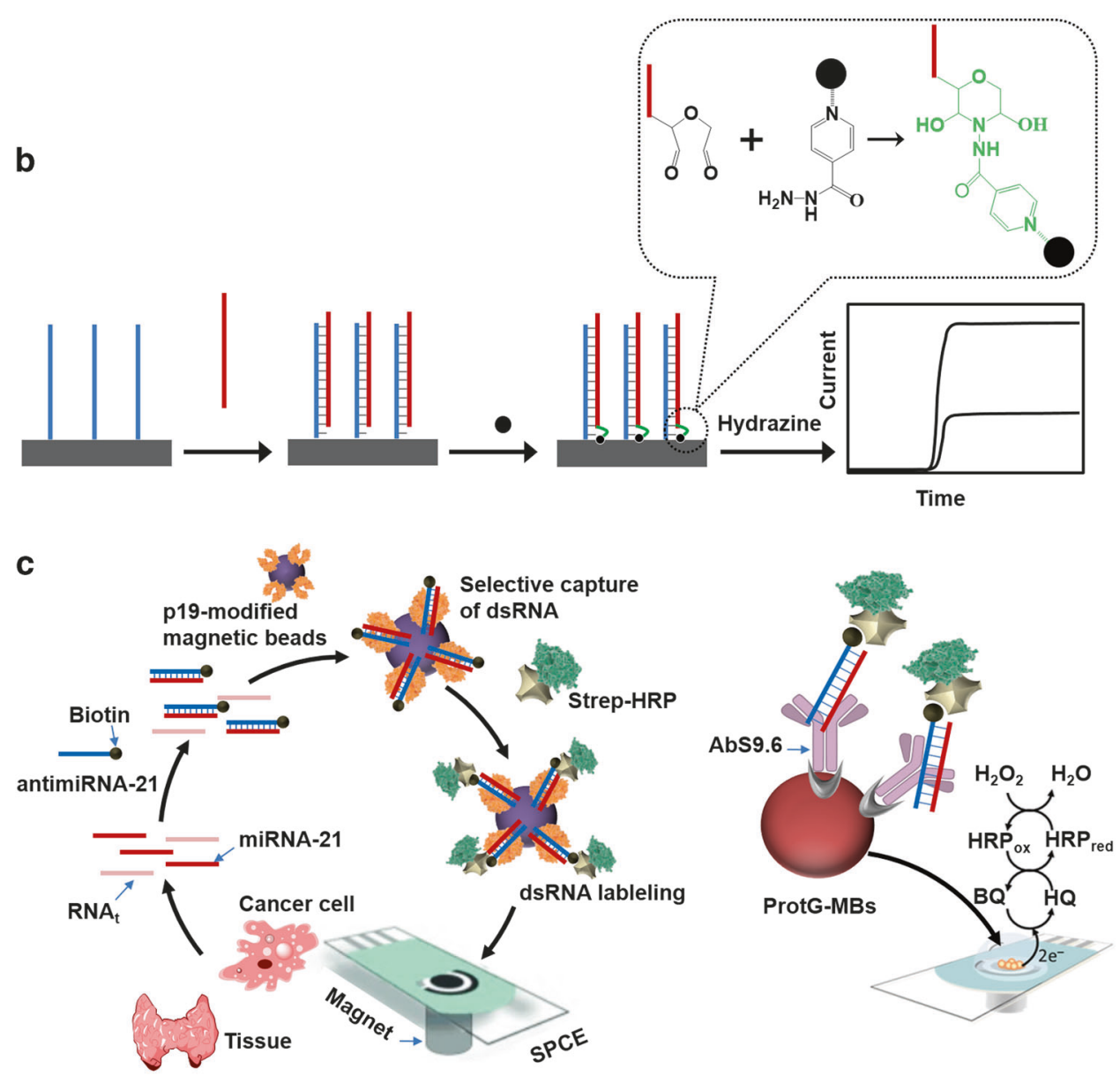

d

AuNPs/GCE conjugated probe DNA
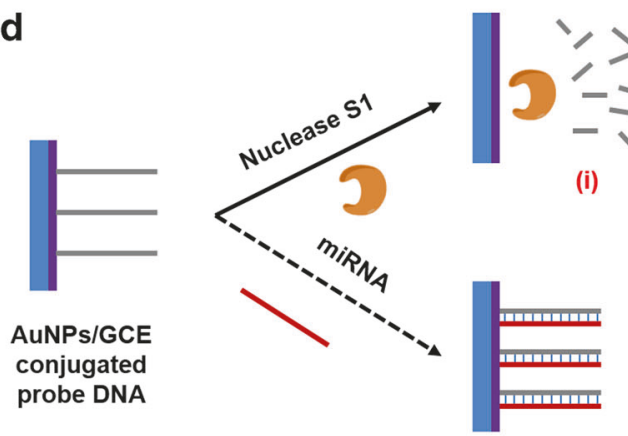

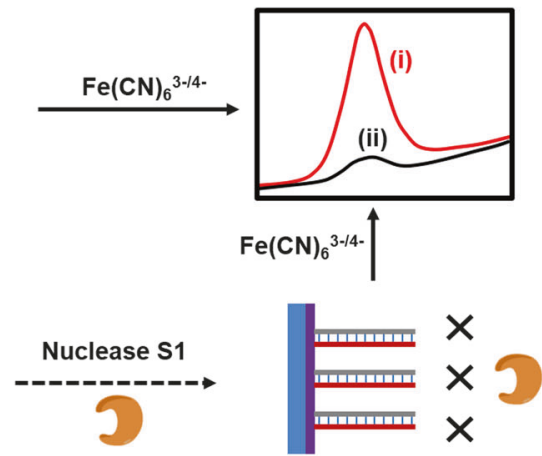

(ii)

bacterial pathogen with a detection limit of $2 \times 10^{2}$ cells $/ \mu \mathrm{L}$ [92]. This device is inspired by the top spinning technology and can be used as a POC device in remote areas. In this device, all the steps from nucleic acid purification, target amplification, and detection have been integrated on the microfluidic disc. The manipulation and mixing of reagents are done by a centrifugal mixing of the pre-loaded reagents. LAMP reaction is used with a low-temperature range from 
a

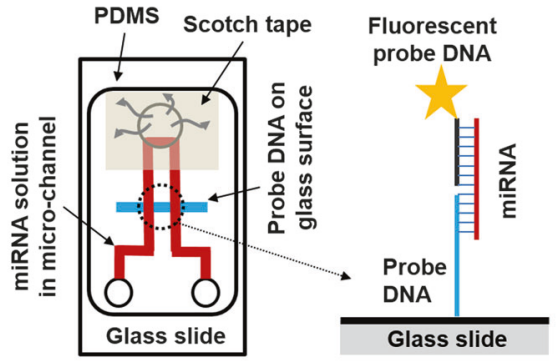

b

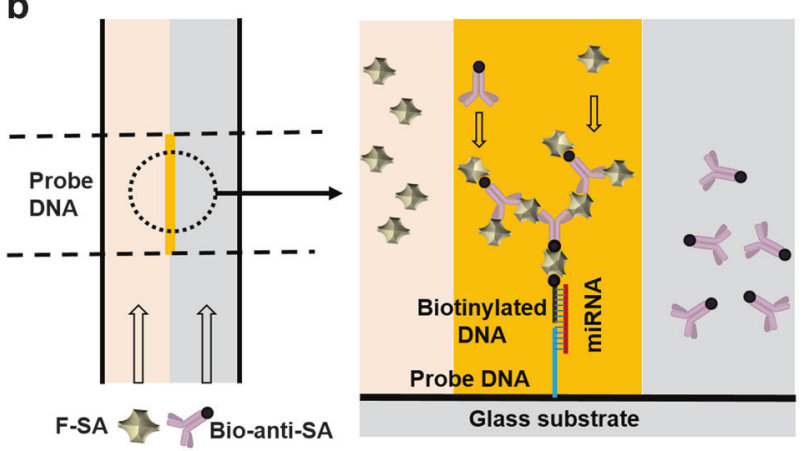

Fig. 6 Microfluidic chip-based approaches. a The power-free microfluidic device in which PDMS absorbs air in the outlet chamber thus being a self-stand pumping device. Probe DNA is immobilized onto the glass surface, and microchannels convey the sample to the probe and miRNA hybridization and detection takes place. b An enlarged view of a laminar flow in the microchannel. The laminar flow conveys F-SA and B-anti-SA. Sandwich hybridization and dendritic amplification take place at the intersection between the probe DNA-patterned surface and the interface of the laminar flow. F-SA FITC-labeled streptavidin, B-anti-SA biotinylated anti-streptavidin. Reproduced with permission from refs. $[90,91]$

30 to $60{ }^{\circ} \mathrm{C}$ through portable pocket warmer-based heating, and a small UV flashlight is used for fluorescence signals visualization. The authors presented the successful detection of $P$. aeruginosa, $S$. typhimurium, and $S$. iniae, as well as the absence of $V$. parahaemolyticus, $V$. vulnificus, and $V$. alginolyticus in artificial urine samples. However, a clinical evaluation of this POC system is not yet performed. It is important to optimize and evaluate the performance of the system and compare with gold standard technologies. Salim et al. took a further step in this direction by using a miRNAbased microfluidic POC system. This device is fully integrated with fluorescence reader. Using this system with specially designed of miRNA beacon probes, it is possible to screen and to detect miRNA-21 in the blood samples of breast cancer patient within $30 \mathrm{~min}$ [93]. The molecular beacon probes were designed to include multiple miRNAs so that multiple miRNA levels in the sample can be detected in a single test to enhance true positive and true negative while eliminating the possibility of other diseases. Using this POC device to test 51 blood samples in which 30 were from healthy, and 21 were from breast cancer patients, miRNA-21 was chosen as the biomarker since it showed four-fold overexpression in the serum of breast cancer patients [93]. The performance of this system was validated against the RT-qPCR. The study revealed that the use of this test could help to depict the stage of cancer, which would be useful for clinician deciding a therapeutic regime. It is very crucial to address the present state of the POC system for clinical diagnosis. Fernández-Carballo et al. were first to report a quantitative and continuous-flow RTPCR-based microfluidic platform for detection of RNAbased virus [94]. This disposable system is suitable for industrial mass production. This developed POC system was used to test and to evaluate against a conventional PCR using a commercial kit to detect Ebola virus. The results of this study showed that the POC system was able to perform faster RT-qPCR with the same analytical sensitivity and efficiency. The amplification and sensitivity are purely depending on the PCR master mixture used, but this system shows fairly high sensitivity in short turnaround time of 30-50 min. This system opens up the possibility of its application for detection of other RNA viruses, such as Zika virus or Chikungunya virus, for remote settings with low medical infrastructure and outbreak control.

\section{Summary and future outlooks}

Over the last decade, the involvement of miRNAs in regulating gene expression has been studied and demonstrated in many diseases $[1,6,7]$. With the advancement of micronano fabrication technologies and life science, the development of POC systems for detection of miRNA as diagnostic and prognostic markers have attracted attention from researchers, clinicians, and industries. The POC diagnostics is a growing field to achieve early diagnostics and better patient outcome [13, 95]. Despite the fast-growing area of POC testing platforms related to antigen-antibody and DNA-based, the development of POC for miRNA detection is still at the very early stage and needs further development and progress. The current strategies for miRNA quantification detection like RT-qPCR, microarray, NGS, NB, and ISH have some limitations to adapt to POC diagnostics. Thus, alternative amplification and novel detection technologies are desirable and on their way to achieve the goals to fit the criteria for POC testing.

The miRNA amplification strategies using the isothermal amplifications are crucial in the development of POC systems and can provide better sensitivity than other existing nucleic acid-based POC systems [64, 65]. However, the small size of miRNAs is a significant obstacle to design primers and probes for development of isothermal amplification technologies, such as RCA or LAMP-based amplification. When designing the RCA system, the specificity of the method is one of the most critical factors since 
template-dependent ligation could lead to non-specific amplification. Thus, optimization of different conditions and careful selection of ligase are required. Similarly in LAMP technology, designing the multiplexing strategies and internal amplification control are challenging. In addition, to develop more sensitive isothermal detection technologies for POC, a miniaturized fluorescent reader is required, and such requirement does indeed imply great technological challenges. Development of portable optical detection system in combination with precise thermal control and innovative isothermal amplification may lead to a new innovative generation of miRNA POC systems for near-site and low resource setting laboratories.

Paper-based POC testing method for the detection of miRNAs holds excellent potential for diagnostics [72, 73], since it is a simple, fast, selective, and sensitive approach for detection of miRNA without any complex sample treatment and expensive instrument. Interestingly, the target miRNA could easily be detected by observing the change of the color and can be quantified by a simple "strip reader" instrument. Further, the signals could easily be enhanced by the use of fluorescent/GNP or enzyme coupled NP. The $\mathrm{pH}$ indicator-based miRNA detection could serve as an ideal on-site POC assay for miRNA diagnostics [74]. However, further studies to include the mismatched detection in miRNA and to improve the capability for multiplexing on the lateral flow strips are required.

Recently, nanotechnology has shown the potential to provide advanced materials and surfaces to address various analytical problems related to miRNA diagnostics. The nanobead-based miRNA detection system can be performed in solutions and thus overcome the design of the lateral flow strip. However, the development of multiplex detection is still a challenge. For futuristic miRNA POC diagnostics, electrochemical signaling-based assay $[80,96]$ could be integrated with the nanotechnology. Lastly, but more critical, miniaturization of miRNA diagnostics assays/device on a small chip using the micro-fluidics could help to develop a comprehensive miRNA POC diagnostics [90, 91].

Despite the many advancements in technologies for miRNA detection, we need to stress a few critical points about POC miRNA diagnostics. The small size and a very minute fraction of target-miRNA in biological samples pose an urgent need for the development of a better miRNA extraction system, an innovative miRNA amplification strategy, and signal enhancement approach in minimal time. Additionally, miRNA sequences may differ in just one to few nucleotides. Thus, the precise design of molecular discrimination is needed. Numerous designs and strategies for miRNA detection have been developed, but they are still at an infant stage for usage in a clinical setting, and further developments are needed to integrate these techniques into POC diagnostics. The in situ detection of multiple miRNAs directly in the biological samples, such as blood, plasma, serum, etc., may serve as an ideal miRNA diagnostic assay and can be integrated into a POC platform to realize fully integrated POC for clinical diagnosis industries in the near future.

Acknowledgements This work has received funding from the European Research Council (ERC) under the European Union's Horizon 2020 research and innovation program (grant agreement no. 68797).

\section{Compliance with ethical standards}

Conflict of interest The authors declare that they have no conflict of interest.

\section{References}

1. Essandoh K, Fan GC. Role of extracellular and intracellular microRNAs in sepsis. Biochim Biophys Acta. 2014;1842: $2155-2162$.

2. Lim LP, Lau NC, Garrett-Engele P, et al. Microarray analysis shows that some microRNAs downregulate large numbers of target mRNAs. Nature. 2005;433:769-773.

3. Calin GA, Croce CM. MicroRNA signatures in human cancers. Nat Rev Cancer. 2006;6:857-866.

4. Wang J, Chen J, Sen S. MicroRNA as biomarkers and diagnostics. J Cell Physiol. 2016;231:25-30.

5. Correia $\mathrm{CN}$, Nalpas NC, McLoughlin KE, et al. Circulating microRNAs as potential biomarkers of infectious disease. Front Immunol. 2017;8:1-17.

6. Chen X, Ba Y, Ma L, et al. Characterization of microRNAs in serum: a novel class of biomarkers for diagnosis of cancer and other diseases. Cell Res. 2008;18282:997-1006.

7. Mitchell PS, Parkin RK, Kroh EM, et al. Circulating microRNAs as stable blood-based markers for cancer detection. Proc Natl Acad Sci USA. 2008;105:10513-10518.

8. Guidelines for microRNA profiling in biofluids. http://www. exiqon.com/ls/Documents/Scientific/microRNA-serum-plasmaguidelines.pdf?

9. Sempere LF. Tissue slide-based microRNA characterization of tumors: how detailed could diagnosis become for cancer medicine? Expert Rev Mol Diagn. 2014;14:853-869.

10. Galimberti D, Villa C, Fenoglia C, et al. Circulating miRNAs as potential biomarkers in alzheimer's disease. J Alzheimers Dis. 2014;42:1261-1267.

11. Weber JA, Baxter DH, Zhang S, et al. The microRNA spectrum in 12 body fluids. Clin Chem. 2010;56:1733-1741.

12. Kost GJ, Tran NK, Louie RF. Point-of-Care Testing: principles, practice, and critical-emergency-disaster medicine. Encycl Anal Chem 2008:1-45.

13. Luppa PB, Bietenbeck A, Beaudoin C, et al. Clinically relevant analytical techniques, organizational concepts for application and future perspectives of point-of-care testing. Biotechnol Adv. 2016;34:139-160.

14. Meyerhans A, Latorre I, Meese E, et al. Micro-RNA-based diagnosis of tuberculosis. European patent application 3026121 A1.2016-06-01.?

15. Zhou M, Yu G, Yang X, et al. Circulating microRNAs as biomarkers for the early diagnosis of childhood tuberculosis infection. Mol Med Rep. 2016;13:4620-4626.

16. Paranjape T, Slack FJ, Weidhaas JB. MicroRNAs: tools for cancer diagnostics. Gut. 2009;58:1546-1554. 
17. Iorio MV, Croce CM. MicroRNA dysregulation in cancer: diagnostics, monitoring and therapeutics. A comprehensive review. EMBO Mol Med. 2012;4:143-159.

18. Price C, Chen J. MicroRNAs in cancer biology and therapy: current status and perspectives. Genes Dis. 2014;1:53-63.

19. D'angelo B, Benedetti E, Cimini A, et al. MicroRNAs: a puzzling tool in cancer diagnostics and therapy. Anticancer Res. 2016;36: $5571-5576$.

20. Cheerla N, Gevaert O. MicroRNA based pan-cancer diagnosis and treatment recommendation. BMC Bioinform. 2017;18:1-11.

21. PDANEWS. Diagnostic test based on MicroRNA technology receives approval. PDANEWS 2008. https://www.fdanews.com/a rticles/108756-diagnostic-test-based-on-microrna-technologyreceives-approval?

22. Gilad S, Lithwick-Yanai G, Barshack I, et al. Classification of the four main types of lung cancer using a microRNA-based diagnostic assay. J Mol Diagn. 2012;14:510-517.

23. Egatz-Gomez A, Wang C, Klacsmann F. Future microfluidic and nanofluidic modular platforms for nucleic acid liquid biopsy in precision medicine. Biomicrofluidics. 2016;10:032902(1)032902(27).

24. Lu J, Getz G, Miska EA, et al. MicroRNA expression profiles classify human cancers. Nature. 2005;435:834-838.

25. Bolha L, Ravnik-Glavac M, Glavac D. Circular RNAs: biogenesis, function, and a role as possible cancer biomarkers. Int $\mathbf{J}$ Genomics. 2017;2017:6218353(1)-6218353(19).

26. Kai K, Dittmar RL, Sen S. Secretory microRNAs as biomarkers of cancer. Semin Cell Dev Biol. 2017;78:22-36.

27. Leggio L, Vivarelli S, L'Episcopo F, et al. microRNAs in Parkinson's disease: from pathogenesis to novel diagnostic and therapeutic approaches. Int J Mol Sci. 2017;18:E2698.

28. Tang G, Reinhart BJ, Bartel DP, et al. A biochemical framework for RNA silencing in plants. Genes Dev. 2003;2:49-63.

29. Böhm-Hofstätter H, Tschernutter M, Kunert R. Comparison of hybridization methods and real-time PCR: their value in animal cell line characterization. Appl Microbiol Biotechnol. 2010;87: $419-425$.

30. Reue K. mRNA quantitation techniques: considerations for experimental design and application. J Nutr. 1998;128:2038-2044.

31. Novogrodsky A, Tal M, Traub A, et al. The and enzymatic phosphorylation deoxyribonucleic acid of ribonucleic acid. J Biol Chem. 1966;241:2933-2943.

32. Hilario E. End labeling procedures: an overview. Mol Biotechnol. 2004;28:77-80.

33. McDougall JK, Dunn AR, Jones KW. In situ hybridization of adenovirus RNA and DNA. Nature. 1972;236:346-348.

34. Nelson PT, Baldwin DA, Kloosterman WP, et al. RAKE and LNA-ISH reveal microRNA expression and localization in RAKE and LNA-ISH reveal microRNA expression and localization in archival human brain. RNA. 2006;12:187-191.

35. Kloosterman WP, Wienholds E, de Bruijn E, et al. In situ detection of miRNAs in animal embryos using LNA-modified oligonucleotide probes. Nat Methods. 2006;3:27-29.

36. Kwon S. Single-molecule fluorescence in situ hybridization: quantitative imaging of single RNA molecules. BMB Rep. 2013;46:65-72.

37. Hanna JA, Wimberly H, Kumar S, et al. Quantitative analysis of microRNAs in tissue microarrays by in situ hybridization. Biotechniques. 2012;52:235-245.

38. Parisi F, Micsinai M, Strino F, et al. Integrated analysis of tumor samples sheds light on tumor heterogeneity. Yale J Biol Med. 2012;85:347-361.

39. Wei Y, Xu F, Li P. Technology-driven and evidence-based genomic analysis for integrated pediatric and prenatal genetics evaluation. J Genet Genom. 2013;40:1-14.
40. Martin CL, Warburton D. Detection of chromosomal aberrations in clinical practice: from karyotype to genome sequence. Annu Rev Genom Hum Genet. 2015;16:309-326.

41. Vedarethinam I, Shah P, Dimaki M, et al. Metaphase FISH on a chip: miniaturized microfluidic device for fluorescence in situ hybridization. Sensors. 2010;10:9831-9846.

42. Kwasny D, Vedarethinam I, Shah P, et al. Advanced microtechnologies for detection of chromosome abnormalities by fluorescent in situ hybridization. Biomed Microdevices. 2012;14: 453-460.

43. Kao KJ, Tai $\mathrm{CH}$, Chang $\mathrm{WH}$, et al. A fluorescence in situ hybridization (FISH) microfluidic platform for detection of HER2 amplification in cancer cells. Biosens Bioelectron. 2015;69: 272-279.

44. Fan JB. Next-generation MicroRNA expression profiling technology: methods and protocols. New York: Humana Press; 2012.

45. Shi R, Chiang VL. Facile means for quantifying microRNA expression by real-time PCR. Biotechniques. 2005;39:519-524.

46. Mei Q, Li X, Meng Y, Wu Z, et al. A facile and specific assay for fuantifying microRNA by an optimized RT-qPCR approach. PLoS ONE. 2012;7:e46890.

47. Ge Q, Tian F, Zhou Y, et al. A universal linker-RT PCR based quantitative method for the detection of circulating miRNAs. Anal Methods. 2014;6:9101-9107.

48. Chen C, Ridzon DA, Broomer AJ, et al. Real-time quantification of microRNAs by stem-loop RT-PCR. Nucleic Acids Res. 2005;33:e179.

49. Raymond CK, Robert BS, Garrett-Engele P, et al. Simple, quantitative primer-extension PCR assay for direct monitoring of microRNAs and short-interfering RNAs. RNA. 2005;11: $1737-1744$.

50. Huang T, Yang J, Liu G, et al. Quantification of mature MicroRNAs using pincer probes and real-time PCR amplification. PLoS ONE. 2015; 10:e120160.

51. Androvic P, Valihrach L, Elling J, et al. Two-tailed RT-qPCR: a novel method for highly accurate miRNA quantification. Nucleic Acids Res. 2017;45:e144.

52. Li J, Yao B, Huang H, et al. Real-time polymerase chain reaction microRNA detection based on enzymatic stem-loop probes ligation. Anal Chem. 2009;81:5446-5451.

53. Tang F, Hajkova P, Barton SC, et al. MicroRNA expression profiling of single whole embryonic stem cells. Nucleic Acids Res. 2006;34:e9.

54. Aikens RS, Agard DA, Sedat JW. Solid-state imagers for microscopy. Methods Cell Biol. 1988;29:291-313.

55. Jiang L, Duan D, Shen Y, et al. Direct microRNA detection with universal tagged probe and time-resolved fluorescence technology. Biosens Bioelectron. 2012;34:291-295.

56. Duan D, Zheng KX, Chen Y, et al. Label-free high-throughput microRNA expression profiling from total RNA. Nucleic Acids Res. 2011;39:e154.

57. Yin JQ, Zhao RC, Morris KV. Profiling microRNA expression with microarrays. Trends Biotechnol. 2008;26:70-76.

58. Lu J, Shen Y, Wu Q, et al. The birth and death of microRNA genes in Drosophila. Nat Genet. 2008;40:351-355.

59. Friedländer MR, Chen W, Adamidi C, et al. Discovering microRNAs from deep sequencing data using miRDeep. Nat Biotechnol. 2008;26:407-415.

60. Diehl P, Fricke A, Sander L, et al. Microparticles: major transport vehicles for distinct microRNAs in circulation. Cardiovasc Res. 2012;93:633-644.

61. Moldovan L, Batte KE, Trgovcich J, et al. Methodological challenges in utilizing miRNAs as circulating biomarkers. J Cell Mol Med. 2014;18:371-390. 
62. Huang J, Borchert GM, Dou D, et al. Bioinformatics in MicroRNA research. New York: Humana Press; 2017.

63. Price CP, John AS, Kricka LJ. Point-of-care testing: needs, opportunity, and innovation. Washington, DC: AACC Press; 2010.

64. Jia H, Li Z, Liu C, et al. Ultrasensitive detection of microRNAs by exponential isothermal amplification. Angew Chem Int Ed Engl. 2010;49:5498-5501.

65. Li C, Li Z, Jia H, et al. One-step ultrasensitive detection of microRNAs with loop-mediated isothermal amplification (LAMP). Chem Commun. 2011;47:2595-2597.

66. Notomi T, Okayama H, Masubuchi H, et al. Loop-mediated isothermal amplification of DNA. Nucleic Acids Res. 2000;28:e63.

67. Jonstrup SP, Koch J, Kjems J. A microRNA detection system based on padlock probes and rolling circle amplification. RNA. 2006;12:1747-1752.

68. Cheng Y, Zhang X, Li Z, et al. Highly sensitive determination of microRNA using target-primed and branched rolling-circle amplification. Angew Chem Int Ed Engl. 2009;48:3268-3272.

69. Goo NI, Kim DE. Rolling circle amplification as isothermal gene amplification in molecular diagnostics. BioChip J. 2016;10: 262-271.

70. Sajid M, Kawde AN, Daud M. Designs, formats and applications of lateral flow assay: a literature review. J Saudi Chem Soc. 2015; 19:689-705.

71. Tripathi P, Upadhyay N, Nara S. Recent advancements in lateral flow immunoassays: a journey for toxin detection in food. Crit Rev Food Sci Nutr. 2018;58:1715-1734.

72. Hou SY, Hsiao YL, Lin MS, et al. MicroRNA detection using lateral flow nucleic acid strips with gold nanoparticles. Talanta. 2012;99:375-379.

73. Gao X, Xu LP, Wu T, et al. An enzyme-amplified lateral flow strip biosensor for visual detection of microRNA-224. Talanta. 2016;146:648-654.

74. Feng C, Mao X, Shi H, et al. Detection of microRNA: a point-ofcare testing method based on a pH-responsive and highly efficient isothermal amplification. Anal Chem. 2017;89:6631-6636.

75. Choi Y, Metcalf G, Sleiman MH, et al. Oligonucleotide-templated reactions based on Peptide Nucleic Acid (PNA) probes: concept and biomedical applications. Bioorg Med Chem. 2014;22: 4395-4398.

76. Metcalf GAD, Shibakawa A, Pate $\mathrm{H}$, et al. Amplification-free detection of circulating microRNA biomarkers from body fluids based on fluorogenic oligonucleotide-templated reaction between engineered peptide nucleic acid probes: application to prostate cancer diagnosis. Anal Chem. 2016;88:8091-8098.

77. Degliangeli F, Pompa PP, Fiammengo R. Nanotechnology-based strategies for the detection and quantification of microRNA. Chemistry. 2014;20:9476-9492.

78. Degliangeli F, Kshirsagar P, Brunetti V, et al. Absolute and sirect microRNA quantification using DNA-gold nanoparticle probes. J Am Chem Soc. 2014;136:2264-2267.

79. Pang Y, Wang C, Wang J, et al. Fe3O4@Ag magnetic nanoparticles for microRNA capture and duplex-specific nuclease signal amplification based SERS detection in cancer cells. Biosens Bioelectron. 2016;79:574-580.
80. Hamidi-asl E, Palchetti I, Hasheminejad E, et al. A review on the electrochemical biosensors for determination of microRNAs. Talanta. 2013;115:74-83.

81. Labuda J, Brett AMO, Evtugyn G, et al. Electrochemical nucleic acid-based biosensors: concepts, terms, and methodology (IUPAC Technical Report). Pure Appl Chem. 2010;82:1161-1187.

82. Gao Z, Yang Z. Detection of microRNAs using electrocatalytic nanoparticle tags. Anal Chem. 2006;78:1470-1477.

83. Campuzano S, Torrente-Rodríguez RM, López-Hernández E, et al. Magnetobiosensors based on viral protein p19 for microRNA determination in cancer cells and tissues. Angew Chem Int Ed Engl. 2014;53:6168-6171.

84. Torrente-Rodríguez RM, Montiel VRV, Campuzano S, et al. Fast electrochemical miRNAs determination in cancer cells and tumor tissues with antibody-functionalized magnetic microcarriers. ACS Sensors. 2016;1:896-903.

85. Vogt VM. Purification and properties of single-strand-specific nuclease from Aspergillus oryzae. Eur J Biochem. 1973;33: 192-200.

86. Zhou Y, Wang M, Yang Z, et al. Electrochemical biosensor for microRNA detection based on hybridization protection against nuclease S1 digestion. J Solid State Electrochem. 2016;20: 413-419.

87. Wang J. Electrochemical biosensors: towards point-of-care cancer diagnostics. Biosens Bioelectron. 2006;21:1887-1892.

88. Whitesides GM. The origins and the future of microfluidics. Nature. 2006;442:368-373.

89. Craighead H. Future lab-on-a-chip technologies for interrogating individual molecules. Nature. 2006;442:387-393.

90. Arata H, Komatsu H, Han A, Hosokawa K, Maeda M. Rapid microRNA detection using power-free microfluidic chip: coaxial stacking effect enhances the sandwich hybridization. Analyst. 2012;137:3234-3237.

91. Arata H, Komatsu H, Hosokawa K. Rapid and sensitive MicroRNA detection with laminar flow-assisted dendritic amplification on power-free microfluidic chip. PLoS ONE. 2012;7: e48329.

92. Zhang L, Tian F, Liu C, et al. Hand-powered centrifugal microfluidic platforms inspired by a spinning top for sample-to-answer diagnostics of nucleic acids. Lab Chip. 2018;18:610-619.

93. Salim B, Athira MV, Kandaswamy A, et al. Microfluidic device for novel breast cancer screening by blood test using miRNA beacon probe. Biomed Micro. 2017;19:89.

94. Fernández-Carballo BL, McBeth $\mathrm{C}$, McGuiness I, et al. Continuous-flow, microfluidic, qRT-PCR system for RNA virus detection. Anal Bioanal Chem. 2018;410:33-43.

95. Vashist SK, Luppa PB, Yeo LY, et al. Emerging technologies for next-generation point-of-care testing. Trends Biotechnol. 2015;33: 692-705.

96. Teo AKL, Lim CL, Gao Z. The development of electrochemical assays for microRNAs. Electrochim Acta. 2014;126:19-30.

97. King KR, Grazette LP, Paltoo DN, et al. Point-of-care technologies for precision cardiovascular care and clinical research: National Heart, Lung, and Blood Institute Working Group. JACC Basic Transl Sci. 2016;1:73-86. 